\title{
Fish consumption and the incidence of cerebrovascular disease
}

\author{
Jukka Montonen $^{1 *}$, Ritva Järvinen ${ }^{2}$, Antti Reunanen ${ }^{1}$ and Paul Knekt ${ }^{1,3}$ \\ ${ }^{1}$ Department of Health and Functional Capacity, National Public Health Institute, Mannerheimintie 166, FIN-00300, Helsinki, \\ Finland \\ ${ }^{2}$ Department of Clinical Nutrition, University of Kuopio, Kuopio, FIN-70211, Finland \\ ${ }^{3}$ Social Insurance Institution, Helsinki, FIN-00100 and Turku, FIN-20720, Finland
}

(Received 14 September 2008 - Revised 23 January 2009 - Accepted 27 January 2009 - First published online 15 April 2009)

Studies of the beneficial role of fish consumption in the prevention of CVD are not consistent in their findings, particularly those studies that focus on the risk of stroke. The aim of the present study is to investigate the relationship between the consumption of different types of fish and the subsequent incidence of cerebrovascular disease (CVA). We prospectively evaluated the association between consumption of different types of fish and CVA in 3958 men and women aged 40-79 years who were free of heart disease and had participated in a health examination survey from 1967 to 1972. A total of 659 incident cases of CVA occurred during a follow-up until the end of 1994. A dietary history interview method provided data on habitual consumption of fish and other foods over the preceding year at baseline. Total fish intake did not predict CVA, but consumption of salted fish suggested an increased risk of intracerebral haemorrhage. The relative risk of intracerebral haemorrhage between the highest tertile of salted fish consumption and non-consumers was 1.98 (95\% CI 1.02, 3.84; $P$ for trend $=0.06$ ) after adjustment for age, sex, energy intake, smoking, BMI, physical activity, geographic area, occupation, diabetes, use of post-menopausal hormones, serum cholesterol, hypertension, and consumptions of butter, vegetables, fruits and berries. The relationship between fish consumption and stroke risk is not straightforward. How the fish is prepared for consumption may play an important role, affecting the association.

Cerebrovascular disease: Stroke: Fish: Salt: Longitudinal studies

Despite the fact that fish is recommended as a part of healthy diet, evidence of fish consumption providing protection against CVD is not entirely consistent, especially when it comes to stroke. The study of this association is also complicated by the different types of stroke, characterized by different risk factors. The meta-analyses of He et al. ${ }^{(1)}$ and Bouzan et al. ${ }^{(2)}$ on fish consumption and stroke risk concluded that there is an inverse relationship between fish consumption and the risk of stroke, the association being concentrated on the ischaemic type of stroke. Another recent careful review suggested that intake of $n-3$ fatty acids specific to fish may reduce the risk of cardiac death and possibly stroke ${ }^{(3)}$. However, in another recent systematic review, the authors did not reach a similar conclusion ${ }^{(4)}$.

It has been suggested that fish consumption may be a potential protective factor when it comes to illnesses because fish contain long-chain $n-3$ fatty acids, which may inhibit atherosclerotic processes ${ }^{(5)}$. On the other hand, the type of fish or method of fish preparation may influence the potential preventive effect. Only a few studies have reported on the association between consumption of different types of fish and stroke, however. In a cohort study among elderly individuals based on the data from the Cardiovascular Health Study, a lower risk of ischaemic stroke was reported with higher consumption of tuna or other broiled or baked fish, while the intake of fried fish or fish sandwiches was associated with a higher risk $^{(6)}$. Salted fish products contain considerable amounts of salt. In ecological studies, salt consumption has been associated with stroke risk $^{(7,8)}$, yet evidence from such prospective studies remains sparse and controversial ${ }^{(9,10)}$. The present study addressed the question of whether an association can be found between total fish consumption, salted or smoked fish in particular, and the subsequent incidence of cerebrovascular disease (CVA), intracerebral haemorrhage or thrombotic stroke in a nationwide cohort of Finns.

\section{Subjects and methods}

The Finnish Mobile Clinic Health Examination Survey carried out health examinations in various parts of Finland between 1966 and $1972^{(11-13)}$. The baseline examinations were carried out in thirty-four rural, industrial or semi-urban populations in six regions of the country. A dietary history interview of 10054 citizens 15 years of age or more was included in the study from $1967^{(14)}$. After exclusion of individuals suffering from heart disease prior to the baseline study, and inclusion of persons aged 40-79 years with an energy intake between 3347 and $25104 \mathrm{~kJ}$ (800 and $6000 \mathrm{kcal})$, the study population was 3958 men and women.

Abbreviation: CVA, cerebrovascular disease.

*Corresponding author: Dr Jukka Montonen, fax +44 35894744 8924, email jukka.montonen@thl.fi 
All participants completed a questionnaire that yielded information on their residence, previous and current illnesses, use of medications, smoking habits and leisure-time physical activity. Subjects were classified according to smoking status as non-smokers, ex-smokers, smokers of pipes or cigars only, smokers of fewer than 15 cigarettes/d, and smokers of 15 or more cigarettes $/ \mathrm{d}^{(12)}$. The participants were asked whether or not they engaged in regular leisure-time physical activity. Body height and weight were measured, and the BMI was calculated. Casual blood pressure was registered in the sitting position after a $5 \mathrm{~min}$ rest, using the auscultatory method $^{(11)}$. The subjects were divided into four classes on the basis of systolic and diastolic blood pressure levels and their use of antihypertensive drugs ${ }^{(12)}$. Persons with systolic blood pressure $\geq 170 \mathrm{mmHg}$ and diastolic blood pressure $\geq 100 \mathrm{mmHg}$, and persons using antihypertensive medication, were considered definitely hypertensive. Persons with systolic blood pressure $\geq 160 \mathrm{mmHg}$ and diastolic blood pressure $\geq 95 \mathrm{mmHg}$ but not defined as hypertensive were considered to have mild hypertension, and those with systolic blood pressure $<140 \mathrm{mmHg}$ and diastolic blood pressure $<90 \mathrm{mmHg}$ were considered normotensive. All persons with intermediate values were considered to have borderline hypertension. Serum cholesterol concentration was determined with an autoanalyser modification of the Liebermann-Burchard reaction $^{(15)}$. Known cases of diabetes were identified by the history given by the participants, and undiagnosed cases were identified on the basis of a modified oral glucose test and fasting blood glucose values, using the current diagnostic criteria of the $\mathrm{WHO}^{(13,16)}$.

Total habitual food consumption during the previous year was estimated using a dietary history interview ${ }^{(14)}$. Trained interviewers conducted these structured interviews using a questionnaire form listing over 100 food items and mixed dishes common in the Finnish diet. Several questions were open-ended, giving the respondent an opportunity to provide more detailed answers and any further explanations during the interview. Food models were used as an aid in determining portion sizes. The consumption of foods was estimated per day, week, month or year according to the choice of the respondent. Individual consumption of food items was converted to g/d. Energy intake was calculated based on the intake of protein, fat and available carbohydrate. Nutrient contents in food items were derived from Finnish food composition tables ${ }^{(17)}$. Food composition data on fatty acids were completed, utilizing analysed values of Finnish foods ${ }^{(18,19)}$.

Reproducibility of the dietary history method was estimated after 4-8 months (ninety-three individuals) and 4-7 years (1844 individuals) by repeating the interview. The reproducibility of the dietary interviews completed 4-8 months apart was good, but long-term consistency 4-7 years apart was rather poor $^{(20)}$. The reliability coefficient for short-term repeatability of the daily consumption of fish was 0.72 , and the corresponding long-term coefficient was $0 \cdot 39$.

Information regarding CVA incidence during the follow-up was obtained by linking data from the Finnish Hospital Discharge Register, kept by the National Board of Health, to the dietary data ${ }^{(21)}$. Information on mortality was based on death certificates obtained for all the deceased from the Central Statistical Office of Finland ${ }^{(13)}$. The diagnosis leading to hospitalization or death due to CVA was identified using the three-digit codes 430-438 of the International Classification of Diseases, Eighth Revision $\left(\mathrm{ICD}-8^{(22)}\right)$. The diagnoses were further classified as intracerebral haemorrhage (code 431 ), thrombotic or embolic occlusion (codes 432-434) or all acute strokes (codes 430-438 and 344) including also subarachnoidal haemorrhage (code 430). The follow-up refers to the period of observation from the time of the baseline examination until onset of the disease, until death or until the end of the observation period (1994), whichever came first.

The Cox proportional hazards model ${ }^{(23)}$ was used to estimate the association between fish consumption and the incidence of CVA. Dietary variables were categorized as quartiles separately among men and women. Intake of salted fish and smoked fish were categorized as non-consumption as a reference and thereafter as tertiles among consumers, due to the high number of non-consumers. Relative risks were estimated for intake categories using the lowest category as the reference. Potential confounding factors including age, sex, energy intake, smoking, BMI, physical activity, geographic area, occupation, diabetes, use of post-menopausal hormones, hypertension and serum cholesterol were adjusted for by modelling. The potential modifying effect of sex, BMI, serum cholesterol and hypertension was studied by including interaction terms in the model. Tests for trends through the quartiles of intake were carried out on the basis of a likelihood ratio test, treating all variables in the model as continuous by entering the quartile medians.

\section{Results}

A total of 659 incident cases of CVA occurred during the follow-up of 28 years (approximately $30 \%$ were identified through death certificates). Of those cases eighty were intracerebral haemorrhages, 364 were thrombotic occlusions or embolia and forty were subarachnoidal haemorrhages. The rest of the cases were unspecified cerebral strokes. Persons who developed CVA tended to be slightly older. They were more likely hypertensive, and they more often had a history of diabetes (Table 1). Distribution of the dietary variables did not show strong differences between incident cases and non-cases.

Most of the baseline non-dietary risk factors were distributed in roughly similar patterns across the quartiles of fish consumption (Table 2). However, persons in the highest quartile of fish consumption were more likely smokers or physically active, and their serum cholesterol level tended to be higher. Persons with higher fish consumption also seemed to consume more vegetables, fruits and berries and less butter than those with lower fish consumption. Fish consumption was closely associated with intake of marine long-chain $n-3$ fatty acids, EPA and DHA and higher intake of energy, mercury and sodium.

Total fish consumption did not predict CVA, intracerebral haemorrhage or thrombotic stroke (Table 3). Categorization of subjects by fish consumption as consumers and non-consumers did not notably alter the results (data not shown). A similar absence of association was observed for intakes of marine long-chain fatty acids, mercury and sodium.

A significantly increased risk of intracerebral haemorrhage was observed when persons in the highest category of consumption of salted fish were compared to non-consumers. The relative risk of intracerebral haemorrhage between 
Table 1. Personal characteristics at baseline in cases of cerebrovascular disease and non-cases (Mean values and standard deviations)

\begin{tabular}{|c|c|c|c|c|}
\hline & \multicolumn{2}{|c|}{ Cases ( $n 659)$} & \multicolumn{2}{|c|}{ Non-cases ( $n$ 3299) } \\
\hline & Mean & SD & Mean & SD \\
\hline Age (years) & $56 \cdot 2$ & 8.68 & $51 \cdot 3$ & 8.68 \\
\hline Sex ( $\%$ of men) & $51 \cdot 1$ & & $52 \cdot 0$ & \\
\hline BMI $\left(\mathrm{kg} / \mathrm{m}^{2}\right)$ & $26 \cdot 9$ & $4 \cdot 12$ & $26 \cdot 4$ & 3.98 \\
\hline Serum cholesterol $(\mathrm{mmol} / \mathrm{l})$ & 7.05 & 1.38 & 6.97 & 1.41 \\
\hline Hypertension $(\%)^{*}$ & \multicolumn{2}{|c|}{$27 \cdot 2$} & \multicolumn{2}{|c|}{$14 \cdot 2$} \\
\hline Diabetes (\%) & \multicolumn{2}{|c|}{3.9} & \multicolumn{2}{|c|}{$2 \cdot 3$} \\
\hline Smoking $(\%) \dagger$ & \multicolumn{2}{|c|}{$30 \cdot 1$} & \multicolumn{2}{|c|}{$31 \cdot 8$} \\
\hline Physically active (\%) & \multicolumn{2}{|c|}{53.8} & \multicolumn{2}{|c|}{$57 \cdot 7$} \\
\hline Use of post-menopausal hormones (\%) & \multicolumn{2}{|c|}{1.0} & \multirow{2}{*}{\multicolumn{2}{|c|}{$1 \cdot 7$}} \\
\hline \multicolumn{3}{|l|}{ Food consumption (g/d) } & & \\
\hline Fish, total & $35 \cdot 2$ & 38.4 & $36 \cdot 3$ & 43.4 \\
\hline Fish, salted & $9 \cdot 60$ & $17 \cdot 6$ & $10 \cdot 2$ & $23 \cdot 4$ \\
\hline Fish, smoked & $5 \cdot 38$ & $14 \cdot 3$ & 4.86 & 11.5 \\
\hline Fish, other than smoked or salted & $20 \cdot 7$ & $27 \cdot 4$ & $20 \cdot 8$ & $27 \cdot 1$ \\
\hline Vegetables & 104 & 83.5 & 106 & $78 \cdot 6$ \\
\hline Fruits and berries & 144 & 134 & 153 & 125 \\
\hline Butter & $47 \cdot 0$ & $29 \cdot 8$ & $46 \cdot 7$ & $28 \cdot 8$ \\
\hline \multicolumn{5}{|l|}{ Daily dietary intake } \\
\hline Energy (kJ) & 10255 & 3448 & 10531 & 3607 \\
\hline Energy (kcal) & 2451 & 824 & 2517 & 862 \\
\hline Marine long-chain $n-3$ fatty acids $(\mathrm{mg}) \ddagger$ & 350 & 328 & 358 & 369 \\
\hline Mercury $(\mu \mathrm{g})$ & 5.48 & 4.80 & $5 \cdot 66$ & $5 \cdot 18$ \\
\hline Sodium (g) & $3 \cdot 14$ & 1.48 & 3.25 & 1.67 \\
\hline
\end{tabular}

* Proportion of persons with systolic blood pressure $\geq 160 \mathrm{mmHg}$ and diastolic $\geq 95 \mathrm{mmHg}$, or having antihypertensive medication. $\dagger$ Proportion of current smokers.

‡EPA + DHA.

Table 2. Mean levels of characteristics in quartiles of fish intake*

(Mean values and standard deviations)

\begin{tabular}{|c|c|c|c|c|c|c|c|c|}
\hline & \multicolumn{8}{|c|}{ Quartile of fish consumption } \\
\hline & \multicolumn{2}{|c|}{1 (lowest) } & \multicolumn{2}{|c|}{2} & \multicolumn{2}{|c|}{3} & \multicolumn{2}{|c|}{4} \\
\hline & Mean & SD & Mean & SD & Mean & SD & Mean & SD \\
\hline Age (years) & $53 \cdot 2$ & $9 \cdot 41$ & 51.9 & $8 \cdot 70$ & $51 \cdot 4$ & $8 \cdot 50$ & $52 \cdot 1$ & $8 \cdot 78$ \\
\hline Sex (\% of men) & $50 \cdot 3$ & & $53 \cdot 0$ & & $52 \cdot 3$ & & $51 \cdot 7$ & \\
\hline BMI $\left(\mathrm{kg} / \mathrm{m}^{2}\right)$ & $26 \cdot 1$ & 3.92 & $26 \cdot 5$ & 4.04 & $26 \cdot 6$ & 4.07 & $26 \cdot 6$ & $4 \cdot 00$ \\
\hline Serum cholesterol (mmol/l) & $6 \cdot 81$ & 1.43 & 6.96 & 1.40 & 7.04 & 1.34 & $7 \cdot 13$ & 1.42 \\
\hline Hypertension (\%)† & \multicolumn{2}{|c|}{$17 \cdot 3$} & \multicolumn{2}{|c|}{$15 \cdot 8$} & \multicolumn{2}{|c|}{$16 \cdot 2$} & \multicolumn{2}{|c|}{$16 \cdot 6$} \\
\hline Diabetes (\%) & \multicolumn{2}{|c|}{2.7} & \multicolumn{2}{|c|}{$2 \cdot 1$} & \multicolumn{2}{|c|}{$2 \cdot 2$} & \multicolumn{2}{|c|}{$3 \cdot 1$} \\
\hline Smoking (\%)‡ & \multicolumn{2}{|c|}{$28 \cdot 5$} & \multicolumn{2}{|c|}{29.9} & \multicolumn{2}{|c|}{$31 \cdot 2$} & \multicolumn{2}{|c|}{$36 \cdot 3$} \\
\hline Physically active (\%) & \multicolumn{2}{|c|}{$50 \cdot 0$} & \multicolumn{2}{|c|}{$56 \cdot 3$} & \multicolumn{2}{|c|}{$59 \cdot 6$} & \multicolumn{2}{|c|}{$62 \cdot 3$} \\
\hline Use of post-menopausal hormones (\%) & \multicolumn{2}{|c|}{$2 \cdot 1$} & \multicolumn{2}{|c|}{$1 \cdot 3$} & \multicolumn{2}{|c|}{$1 \cdot 1$} & \multicolumn{2}{|c|}{1.6} \\
\hline \multicolumn{9}{|l|}{ Food consumption $(\mathrm{g} / \mathrm{d})$} \\
\hline Fish, salted & 1.51 & $2 \cdot 26$ & $4 \cdot 48$ & $4 \cdot 71$ & 8.90 & $9 \cdot 29$ & $25 \cdot 3$ & $39 \cdot 5$ \\
\hline Fish, smoked & 0.53 & 1.44 & $2 \cdot 37$ & $3 \cdot 76$ & $5 \cdot 00$ & $6 \cdot 79$ & $13 \cdot 2$ & $24 \cdot 6$ \\
\hline Fish, other than smoked or salted & 4.09 & 3.37 & 11.4 & $5 \cdot 79$ & $20 \cdot 0$ & $10 \cdot 8$ & $47 \cdot 4$ & $41 \cdot 1$ \\
\hline Vegetables & $81 \cdot 2$ & $71 \cdot 1$ & 103 & $74 \cdot 3$ & 114 & 76.9 & 125 & $87 \cdot 9$ \\
\hline Fruits and berries & 128 & 121 & 149 & 115 & 154 & 124 & 175 & 141 \\
\hline Butter & $44 \cdot 2$ & $28 \cdot 1$ & $45 \cdot 5$ & $28 \cdot 2$ & $46 \cdot 5$ & $28 \cdot 0$ & $50 \cdot 7$ & $31 \cdot 0$ \\
\hline \multicolumn{9}{|l|}{ Daily dietary intake } \\
\hline Energy (kJ) & 9540 & 3276 & 10259 & 3469 & 10657 & 3414 & 11481 & 3858 \\
\hline Energy (kcal) & 2280 & 783 & 2452 & 829 & 2547 & 816 & 2744 & 922 \\
\hline Marine long-chain $n-3$ fatty acids $(\mathrm{mg}) \S$ & 124 & 75.9 & 222 & 110 & 350 & 149 & 728 & 520 \\
\hline Mercury $(\mu \mathrm{g})$ & $2 \cdot 60$ & 0.88 & $3 \cdot 82$ & $1 \cdot 26$ & $5 \cdot 33$ & 1.97 & $10 \cdot 7$ & $7 \cdot 70$ \\
\hline Sodium (g) & 2.57 & $1 \cdot 12$ & $2 \cdot 91$ & $1 \cdot 19$ & $3 \cdot 25$ & $1 \cdot 27$ & $4 \cdot 20$ & $2 \cdot 22$ \\
\hline
\end{tabular}

*For details of subjects and procedures, see the Subjects and methods section and Table 1.

$\dagger$ Persons with systolic blood pressure $\geq 160 \mathrm{mmHg}$ and diastolic $\geq 95 \mathrm{mmHg}$, or having antihypertensive medication.

† Proportion of current smokers.

$\S E P A+D H A$. 
Table 3. Adjusted relative risks (RR) of cerebrovascular diseases in quartiles of fish consumption and intakes of marine long-chain $n$-3 fatty acids, mercury and sodium*

\begin{tabular}{|c|c|c|c|c|c|c|c|c|c|}
\hline \multirow[b]{2}{*}{ Quartiles of intake (median g/d) } & \multicolumn{3}{|c|}{ Cerebrovascular disease } & \multicolumn{3}{|c|}{ Intracerebral haemorrhage } & \multicolumn{3}{|c|}{ Thrombosis or embolia } \\
\hline & No. of cases & $\mathrm{RR}$ & $95 \% \mathrm{Cl}$ & No. of cases & $\mathrm{RR}$ & $95 \% \mathrm{Cl}$ & No. of cases & $\mathrm{RR}$ & $95 \% \mathrm{Cl}$ \\
\hline \multicolumn{10}{|l|}{ Fish, total } \\
\hline $1(6)$ & 175 & 1.00 & & 19 & 1.00 & & 101 & 1.00 & \\
\hline $2(18)$ & 162 & 1.02 & $0 \cdot 82,1 \cdot 26$ & 18 & 1.00 & $0.51,1.96$ & 91 & 1.05 & $0.78,1.40$ \\
\hline $3(32)$ & 162 & 1.07 & $0.86,1.34$ & 22 & 1.30 & $0.68,2.48$ & 88 & 1.04 & $0.77,1.39$ \\
\hline $4(72)$ & 160 & 1.01 & $0 \cdot 81,1 \cdot 27$ & 21 & $1 \cdot 23$ & $0.63,2.42$ & 84 & 0.99 & $0.73,1.35$ \\
\hline$P$ value for trend & & & 0.80 & & & 0.41 & & & 0.96 \\
\hline \multicolumn{10}{|l|}{ Fish, salted $\dagger$} \\
\hline $1(0)$ & 180 & 1.00 & & 15 & 1.00 & & 102 & 1.00 & \\
\hline $2(2)$ & 163 & 1.06 & $0.86,1.32$ & 22 & 1.47 & $0.75,2 \cdot 88$ & 95 & 1.09 & $0.82,1.45$ \\
\hline $3(6)$ & 152 & 1.08 & $0 \cdot 86,1 \cdot 34$ & 18 & 1.32 & $0.65,2.67$ & 85 & 1.05 & $0.78,1.41$ \\
\hline $4(21)$ & 164 & 1.08 & $0 \cdot 86,1 \cdot 34$ & 25 & 1.98 & $1 \cdot 02,3 \cdot 84$ & 82 & 0.97 & $0.72,1.30$ \\
\hline$P$ value for trend & & & 0.44 & & & 0.06 & & & $0 \cdot 82$ \\
\hline \multicolumn{10}{|l|}{ Fish, smoked† } \\
\hline $1(0)$ & 397 & 1.00 & & 42 & 1.00 & & 221 & 1.00 & \\
\hline $2(2)$ & 93 & 0.96 & $0 \cdot 76,1 \cdot 21$ & 16 & 1.56 & $0.86,2 \cdot 83$ & 44 & 0.82 & $0.59,1.14$ \\
\hline $3(7)$ & 85 & 0.92 & $0.72,1.17$ & 12 & $1 \cdot 15$ & $0.59,2.26$ & 47 & 0.87 & $0.63,1.20$ \\
\hline $4(19.5)$ & 84 & 0.92 & $0.72,1 \cdot 17$ & 10 & 0.99 & $0.48,2.06$ & 52 & 1.03 & $0.76,1.41$ \\
\hline$P$ value for trend & & & 0.39 & & & 0.84 & & & 0.81 \\
\hline \multicolumn{10}{|l|}{ Fish, other than smoked or salted } \\
\hline $1(2.5)$ & 168 & 1.00 & & 20 & 1.00 & & 93 & 1.00 & \\
\hline $2(9)$ & 167 & 1.01 & $0 \cdot 81,1 \cdot 26$ & 21 & 0.93 & $0.49,1.75$ & 95 & $1 \cdot 13$ & $0.84,1.52$ \\
\hline $3(17)$ & 157 & 0.93 & $0 \cdot 74,1 \cdot 16$ & 22 & 0.93 & $0.49,1.77$ & 79 & 0.92 & $0.67,1 \cdot 25$ \\
\hline $4(40)$ & 167 & 1.01 & $0.81,1.27$ & 17 & 0.86 & $0.44,1.68$ & 97 & $1 \cdot 12$ & $0.83,1.51$ \\
\hline$P$ value for trend & & & 0.90 & & & 0.69 & & & 0.77 \\
\hline \multicolumn{10}{|l|}{ Marine long-chain $n-3$ fatty acids $\ddagger$} \\
\hline $1(102)$ & 187 & 1.00 & & 17 & 1.00 & & 106 & 1.00 & \\
\hline $2(202)$ & 157 & 0.97 & $0 \cdot 78,1 \cdot 21$ & 21 & 1.20 & $0.61,2.34$ & 85 & 0.95 & $0.71,1 \cdot 28$ \\
\hline 3 (332) & 151 & 0.91 & $0.73,1.14$ & 20 & 1.28 & $0.65,2.53$ & 92 & 0.99 & $0.74,1.33$ \\
\hline $4(655)$ & 164 & 1.01 & $0 \cdot 80,1 \cdot 28$ & 22 & 1.56 & $0.77,3.15$ & 81 & 0.91 & $0.66,1.26$ \\
\hline$P$ value for trend & & & 0.90 & & & 0.22 & & & 0.66 \\
\hline \multicolumn{10}{|l|}{ Mercury§ } \\
\hline $1(2 \cdot 22)$ & 165 & 1.00 & & 18 & 1.00 & & 94 & 1.00 & \\
\hline $2(3.46)$ & 180 & $1 \cdot 16$ & $0.93,1.45$ & 20 & 1.00 & $0.52,1.93$ & 96 & $1 \cdot 18$ & $0.87,1.58$ \\
\hline $3(5 \cdot 08)$ & 156 & 1.06 & $0.84,1.35$ & 25 & $1 \cdot 16$ & $0.60,2.25$ & 92 & $1 \cdot 17$ & $0.85,1.60$ \\
\hline $4(9.31)$ & 158 & $1 \cdot 15$ & $0.90,1.47$ & 17 & 0.99 & $0.47,2.06$ & 82 & $1 \cdot 17$ & $0.84,1.63$ \\
\hline$P$ value for trend & & & 0.40 & & & 0.91 & & & 0.40 \\
\hline \multicolumn{10}{|l|}{ Sodium } \\
\hline $1(1.76)$ & 190 & 1.00 & & 19 & 1.00 & & 107 & 1.00 & \\
\hline $2(2.71)$ & 163 & 0.97 & $0.78,1.21$ & 24 & 1.41 & $0.73,2.69$ & 91 & 0.97 & $0.72,1.30$ \\
\hline $3(3.54)$ & 146 & 0.89 & $0.70,1.14$ & 15 & 0.94 & $0.44,2.03$ & 82 & 0.89 & $0.64,1.23$ \\
\hline $4(4.85)$ & 160 & 1.05 & $0.79,1.40$ & 22 & 1.42 & $0.61,3.30$ & 84 & 0.93 & $0.63,1.38$ \\
\hline$P$ value for trend & & & 0.996 & & & 0.68 & & & 0.61 \\
\hline
\end{tabular}

${ }^{*}$ For details of subjects and procedures, see Subjects and Methods section and Table 1. RR adjusted for age (continuous), sex, energy intake (continuous), smoking (non-smokers, ex-smokers, smokers of pipes or cigars only, smokers of fewer than fifteen cigarettes $/ \mathrm{d}$, and smokers of fifteen or more cigarettes $/ \mathrm{d}), \mathrm{BMI}\left(\mathrm{kg} / \mathrm{m}^{2}:<23,23-24.9\right.$, $25-27.4,27.5-29.9,30+$ ), physical activity (inactive, occasionally or regularly active), geographic area (six areas), occupation, diabetes, use of post-menopausal hormones (yes/no), hypertension (four categories according to measured systolic and diastolic pressure and use of antihypertensive medication), serum cholesterol (continuous), and consumptions of butter, vegetables, fruits and berries (continuous).

$\dagger$ Categorized as non-consumers as reference and as tertiles among consumers due to high number of non-consumers.

$\ddagger E P A+D H A(m g / d)$.

$\S \mu \mathrm{g} / \mathrm{d}$.

non-consumers and the extreme consumption tertile was 1.98 (95\% CI 1.02, 3.84; $P$ for trend: $P=0.06$ ) when adjusted for age, sex, energy intake, smoking, BMI, physical activity, geographic area, occupation, diabetes, use of post-menopausal hormones, serum cholesterol, hypertension, consumptions of butter, vegetables, fruits and berries. Adjustment for sodium intake attenuated the association observed. The relative risk of intracerebral haemorrhage in the extreme category of salted fish was 1.70 (CI $0.84,3.43 ; P=0.19)$ after further adjustment for sodium intake.

The interaction between total fish consumption and the potentially modifying factors of sex, BMI, serum cholesterol and hypertension in the prediction of CVA was studied.
The association between salted fish intake and the risk of intracerebral haemorrhage was more pronounced among normotensive persons and persons with higher serum cholesterol levels (Table 4). Among the normotensive persons, the relative risks across the consumption categories compared to non-consumers were $2.36(95 \%$ CI $1.03,5.55), 1.53(95 \%$ CI $0.62,3.77)$ and $3.16(95 \%$ CI $1.39,7.20)$, and among persons with hypertension 0.31 (95\% CI 0.06, 1.54), 1.17 (95\% CI $0.37,3.78)$ and $0.42(95 \%$ CI $0.09,2 \cdot 05)$, respectively. The $P$ value for interaction was 0.02 . The corresponding risks among persons with median or higher serum cholesterol level were 0.96 (95\% CI $0.31,2.98), 0.86$ (95\% CI 0.26 , $2.83)$ and $2.87(95 \%$ CI 1.12, 7.35), and among persons 
Table 4. Relative risk (RR) of intracerebral haemorrhages between the highest and lowest categories of consumption of fish and salted fish in strata of potential effect-modifying factors*

\begin{tabular}{|c|c|c|c|c|}
\hline & \multicolumn{2}{|c|}{ Fish, total } & \multicolumn{2}{|c|}{ Salted fish† } \\
\hline & $\mathrm{RR}$ & $95 \% \mathrm{Cl}$ & $\mathrm{RR}$ & $95 \% \mathrm{Cl}$ \\
\hline \multicolumn{5}{|l|}{ Sex } \\
\hline Men & 1.63 & $0.67,4.01$ & 2.08 & $0.88,4.88$ \\
\hline Women & 0.88 & $0.33,2.33$ & 1.82 & $0.64,5 \cdot 18$ \\
\hline$P$ for interaction & 0.37 & & & \\
\hline \multicolumn{5}{|l|}{ BMI } \\
\hline Below medianł & 1.51 & $0.60,3.77$ & $2 \cdot 81$ & $1.04,7.59$ \\
\hline Median or higher & $1 \cdot 18$ & $0.48,2.90$ & 1.49 & $0.65,3.41$ \\
\hline$P$ for interaction & 0.30 & & 0.27 & \\
\hline \multicolumn{5}{|l|}{ Serum cholesterol } \\
\hline Below median§ & 0.82 & $0.34,1.97$ & 1.30 & $0.46,3.64$ \\
\hline Median or higher & $2 \cdot 38$ & $0.75,7.55$ & $2 \cdot 87$ & $1 \cdot 12,7 \cdot 35$ \\
\hline$P$ for interaction & 0.44 & & 0.04 & \\
\hline \multicolumn{5}{|l|}{ Hypertension } \\
\hline No & 1.33 & $0.65,2.72$ & 3.16 & $1.39,7.20$ \\
\hline Yes\| & 0.66 & $0.11,4.06$ & 0.42 & $0.09,2.05$ \\
\hline$P$ for interaction & 0.18 & & 0.02 & \\
\hline
\end{tabular}

${ }^{*}$ For details of subjects and procedures, see the Subjects and methods section and Table 1. Relative risk adjusted for age, sex, energy intake, smoking, BMI, physical activity, geographic area, occupation, diabetes, use of post-menopausa hormones, blood pressure, serum cholesterol, and consumptions of butter, vegetables, fruits and berries except when included in the interaction term.

†Categorized as non-consumers as reference and as tertiles among consumers due to high number of non-consumers.

‡ Median value was $26 \cdot 1 \mathrm{~kg} / \mathrm{m}^{2}$

$\S$ Median value was $6.9 \mathrm{mmol} / \mathrm{l}$.

\|Persons with systolic blood pressure $\geq 160 \mathrm{mmHg}$ and diastolic $\geq 95 \mathrm{mmHg}$, or having antihypertensive medication.

with below median serum cholesterol values $2 \cdot 12(95 \% \mathrm{CI}$ $0.89,5.07), 1.90(95 \%$ CI $0.77,4.73)$ and $1.30(95 \% \mathrm{CI}$ $0.46,3.64)$. The $P$ value for interaction was $0 \cdot 04$. No significant interactions between any of these modifying factors and fish consumption were noted in prediction of either total CVA or thrombotic strokes (data not shown).

\section{Discussion}

We did not find a significant association between total fish consumption and the risk of thrombotic or haemorrhagic stroke or total stroke risk. However, the present findings indicated that consumption of salted fish was associated with a higher risk of haemorrhagic stroke. The present findings support the hypothesis that the effects of fish intake on health may depend on the preparation of the fish, especially salting.

Few previous studies have reported on the association between fish consumption and haemorrhagic stroke. No significant associations have been reported among men ${ }^{(24)}$, women $^{(25)}$ or elderly people ${ }^{(6)}$ in the USA whereas a largescale population-based cohort study in Japan suggested that fish consumption, among some other animal products, may reduce the risk of intracerebral haemorrhage ${ }^{(26)}$. He et al. ${ }^{(1)}$ did not observe any significant association between fish consumption and haemorrhagic stroke risk in their meta-analysis on three large cohort studies with data on stroke subtypes.

The method of fish preparation has been considered only in a few studies. Mozaffarian et al. ${ }^{(6)}$ reported a lower risk of ischaemic stroke with higher consumption of tuna or other broiled or baked fish, while intake of fried fish or fish sandwiches was associated with a higher risk of stroke; neither type of fish was associated with haemorrhagic stroke, however. In the present study, salted fish, which was associated with a higher risk of haemorrhagic stroke, represents a high-salt food ${ }^{(27)}$. In the present study, consumption of salted fish was correlated with sodium intake (energyadjusted Pearson correlation coefficient 0.77; $P<0.001$ ), and the association observed attenuated after further adjustment for sodium intake. Therefore we suggest that sodium in these fish products may be a factor increasing stroke risk, but it cannot be ruled out that other factors related to higher salted fish consumption may explain the higher stroke risk. High sodium intake is a known predictor for increased blood pressure ${ }^{(28,29)}$ and hypertension is one of the major risk factors for stroke ${ }^{(30)}$, even more so for haemorrhagic stroke ${ }^{(31)}$. Ecological comparisons between countries and populations indicate a direct relationship between salt consumption and stroke risk ${ }^{(7,8)}$, however, evidence from prospective studies is sparse and controversial ${ }^{(9,10)}$.

In the present study, we were able to estimate the sodium levels in natural foods and processed foods such as cheese, sausages and cured meat, salted and smoked fish, and the salt added in bread. Salt used in home cooking or added at the table was not recorded. It has been estimated from the Finnish Mobile Clinic cohort that in the 1970s, total salt consumption was approximately $14 \mathrm{~g} / \mathrm{d}$ among men and approximately $10 \mathrm{~g} / \mathrm{d}$ among women ${ }^{(27)}$. The most important foods contributing to total salt intake were baked products, mostly bread, sausages, and salted and smoked fish, which accounted for 12 and $11 \%$, respectively, of the total salt intake of men and women ${ }^{(27)}$. Although the contribution of salted fish to the total salt intake was not very high on average, consumption of salted fish may indicate a preference for salty foods.

In the present study, the association between salted fish and haemorrhagic stroke was more pronounced among persons with normal blood pressure, suggesting that as a transcendent risk factor of stroke, hypertension masks the effect of salted fish. On the other hand, the association observed was also more pronounced among persons with serum cholesterol values over the median. However, the serum cholesterol level itself was modestly associated with reduced risk of stroke mortality in a large meta-analysis of sixty-one prospective studies ${ }^{(32)}$.

No significant associations were found between total fish consumption and the risk of total stroke or thrombotic or haemorrhagic stroke. Similarly, in several previous studies total fish consumption was not found to be significantly associated with stroke risk ${ }^{(33-37)}$. However, in their meta-analysis $\mathrm{He}$ et al. ${ }^{(1)}$ and Bouzan et al. ${ }^{(2)}$ concluded that consumption of fish may offer protection against stroke, particularly ischaemic stroke. Even very small amounts of fish, one fish meal per month, were suggested to offer protection against ischaemic stroke $^{(1)}$. In the present study, the majority of the subjects in the lowest quartile reached that level. However, we found no significant association between fish consumption and stroke even when the non-consumers of fish were compared with those with any consumption of fish at all.

A few previous studies have associated a lower risk of stroke, particularly thrombotic stroke, with a higher intake of fish-specific fatty acids ${ }^{(24,25)}$. In two recent systematic 
reviews, the evidence of the benefits of $n$-3 long-chain fatty acids against stroke events was, however, considered to be inconclusive $^{(3,4)}$. A recent study based on an analysis of fatty acids in the blood by Wennberg et al. ${ }^{(38)}$ did not conclude that fish-specific fatty acids would decrease stroke risk. Similarly, no significant association between marine long-chain $n$-3 fatty acids and stroke risk was observed in the present study. In Finland, the type of fish consumed has been mainly low-fat fish. Furthermore, the majority of highfat fish consumed among the study population was salted herring, also contributing to salt and sodium intake. No significant association was found between stroke risk and dietary mercury in the present study, which is in line the results of Wennberg et al. ${ }^{(38)}$, who reported no association between mercury analysed in erythrocytes and stroke risk.

In addition to chance, several methodological issues may have influenced the present results. The dietary history method has its limitations, and the consequent misclassification of subjects tends to alter observable associations between dietary exposure and outcome ${ }^{(39)}$. In the present study, all the interviewers were trained nutrition professionals, and a preformed questionnaire was used to diminish differences between interviewers. The questions were open-ended and offered opportunities to specify the answers. Likewise, food models were used to reduce errors of recall. Our prospective study design minimized the possibility that changes in diet due to prevalent disease influenced the findings. Furthermore, it is unlikely that the current discussion about the potential health effects of fish consumption has interfered with the reported fish consumption, since reports on the potential health effects did not become available until the 1980s. It is possible that a lack of information on the cases because of incomplete information on the incident cerebral infarction during the follow-up ${ }^{(21)}$ and the emigration of some persons during the follow-up period may have caused modestly conservative risk estimates.

In general, the short-term repeatability of the dietary history method was favourable, but long-term reliability was relatively poor ${ }^{(20)}$. The poorer long-term reliability can be explained by changes in Finnish food consumption. During the long follow-up, dietary habits may have changed and attenuated the associations observed.

In conclusion, the present findings clearly demonstrate that the relationship between fish consumption and stroke risk is not straightforward. How the fish is prepared for consumption may be an important factor affecting the association, but it may also depend on which type of stroke is being investigated.

\section{Acknowledgements}

The authors state that there are no conflicts of interest. This research received no specific grant from any funding agency in the public, commercial or not-for-profit sectors. J. M. participated in the analysis and interpretation of data and in the drafting of the manuscript. R. J. collaborated on the interpretation of the results, and participated in drafting the manuscript. P. K. contributed to the development of the analysis plan and was closely involved with the interpretation of the results and editing of the manuscript. He also supervised the conducting of the study. A. R. provided significant consultation on the interpretation of the results and editing of the manuscript.

\section{References}

1. He K, Song Y, Daviglus ML, et al. (2004) Fish consumption and incidence of stroke: a meta-analysis of cohort studies. Stroke 35, 1538-1542.

2. Bouzan C, Cohen JT, Connor WE, et al. (2005) A quantitative analysis of fish consumption and stroke risk. Am J Prev Med 29, $347-352$

3. Wang C, Harris WS, Chung M, et al. (2006) N-3 fatty acids from fish or fish-oil supplements, but not $\alpha$-linolenic acid, benefit cardiovascular disease outcomes in primary- and secondaryprevention studies: a systematic review. Am J Clin Nutr 84, $5-17$.

4. Hooper L, Thompson RL, Harrison RA, et al. (2006) Risks and benefits of omega 3 fats for mortality, cardiovascular disease, and cancer: systematic review. $\mathrm{Br}$ Med J 332, 752-760.

5. Robinson JG \& Stone NJ (2006) Antiatherosclerotic and antithrombotic effects of omega-3 fatty acids. Am J Cardiol 98 $39-49$.

6. Mozaffarian D, Longstreth WT Jr, Lemaitre RN, et al. (2005) Fish consumption and stroke risk in elderly individuals: the Cardiovascular Health Study. Arch Intern Med 165, 200-206.

7. Sasaki S, Zhang X-H \& Kesteloot H (1995) Dietary sodium, potassium, saturated fat, alcohol, and stroke mortality. Stroke 26, 783-789.

8. Xie JX, Sasaki S, Joossens JV, et al. (1992) The relationship between urinary cations obtained from the INTERSALT study and cerebrovascular mortality. J Hum Hypertens 6, 17-21.

9. Cohen HW, Hailpern SM, Fang J, et al. (2006) Sodium intake and mortality in the NHANES II Follow-up Study. Am J Med 119, 275.e277-275.e214.

10. Nagata C, Takatsuka N, Shimizu N, et al. (2004) Sodium intake and risk of death from stroke in Japanese men and women. Stroke 35, 1543-1547.

11. Aromaa A (1981) Epidemiology and Public Health Impact of High Blood Pressure in Finland. Publication of the Social Insurance Institution, AL:17. Helsinki: Social Insurance Institution (in Finnish with an English summary).

12. Knekt P (1988) Serum Alpha-tocopherol and the Risk of Cancer. Publication of the Social Insurance Institution, ML:83. Helsinki: Social Insurance Institution.

13. Reunanen A, Aromaa A, Pyörälä K, et al. (1983) The social insurance institution's coronary heart disease study. Baseline data and 5-year mortality experience. Acta Med Scand Suppl 673, $1-120$.

14. Järvinen R (1996) Epidemiological Follow-up Study on Dietary Antioxidant Vitamins. Results from the Finnish Mobile Clinic Health Examination Survey. Studies in Social Security and Health. no 11. Helsinki: Social Insurance Institution.

15. Huang TC, Chen CP, Wefler V, et al. (1961) A stable reagent for the Lieberman-Burchard reaction. Application to rapid serum cholesterol determination. Anal Chem 33, 1405-1507.

16. World Health Organization (1985) Diabetes Mellitus: Report of a WHO Study Group. Geneva: WHO.

17. Rastas M, Seppänen R, Knuts L-R, et al. (1989) Nutrient Composition of Foods. Helsinki: Social Insurance Institution.

18. Hyvönen L \& Koivistoinen P (1994) Fatty acid analysis, tag equivalents as net fat value, and nutritional attributes of fish fish products. J Food Composition Anal 7, 44-58.

19. Hyvönen L, Lampi A-M, Varo P, et al. (1993) Fatty acid analysis, tag equivalents as net fat values, and nutritional attributes of commercial fats and oils. J Food Composition Anal 6, 24-40.

20. Järvinen R, Seppänen R \& Knekt P (1993) Short-term and longterm reproducibility of dietary history interview data. Int J Epidemiol 22, 520-527.

21. Heliövaara M, Reunanen A, Aromaa A, et al. (1984) Validity of hospital discharge data in a prospective epidemiological study 
on stroke and myocardial infarction. Acta Med Scand 216, $309-315$

22. World Health Organization (1967-9) International Classification of Diseases. Manual of International Statistical Classification of Diseases, Injuries, and Causes of Death, 8th rev. Geneva: WHO.

23. Cox D (1972) Regression models and life-tables. J R Stat Soc B 34, 187-220.

24. He K, Rimm EB, Merchant A, et al. (2002) Fish consumption and risk of stroke in men. JAMA 288, 3130-3136.

25. Iso H, Rexrode KM, Stampfer MJ, et al. (2001) Intake of fish and omega- 3 fatty acids and risk of stroke in women. JAMA 285, 304-312.

26. Sauvaget C, Nagano J, Allen N, et al. (2003) Intake of animal products and stroke mortality in the Hiroshima/Nagasaki Life Span Study. Int J Epidemiol 32, 536-543.

27. Pietinen $\mathrm{P}$ (1981) Sources of sodium in the Finnish diet. $J$ Sci Agric Soc Finland 53, 275-284.

28. Chobanian AV \& Hill M (2000) National heart, lung, and blood institute workshop on sodium and blood pressure: a critical review of current scientific evidence. Hypertension 35, $858-863$

29. Karppanen H \& Mervaala E (2006) Sodium intake and hypertension. Prog Cardiovasc Dis 49, 59-75.

30. Prospective Studies Collaboration (2002) Age-specific relevance of usual blood pressure to vascular mortality: a meta-analysis of individual data for one million adults in 61 prospective studies. Lancet 360, 1903-1913.
31. Song YM, Sung J, Lawlor DA, et al. (2004) Blood pressure, haemorrhagic stroke, and ischaemic stroke: the Korean national prospective occupational cohort study. Br Med J 328, 324-325.

32. Prospective Studies Collaboration (2007) Blood cholesterol and vascular mortality by age, sex, and blood pressure: a meta-analysis of individual data from 61 prospective studies with 55000 vascular deaths. Lancet 370, 1829-1839.

33. Bravata DM, Wells CK, Brass LM, et al. (2007) Dietary fish or seafood consumption is not related to cerebrovascular disease risk in twin veterans. Neuroepidemiology 28, 186-190.

34. Morris MC, Manson JE, Rosner B, et al. (1995) Fish consumption and cardiovascular disease in the Physicians' Health Study: a prospective study. Am J Epidemiol 142, 166-175.

35. Myint PK, Welch AA, Bingham SA, et al. (2006) Habitual fish consumption and risk of incident stroke: the European Prospective Investigation into Cancer (EPIC) Norfolk prospective population study. Public Health Nutr 9, 882-888.

36. Orencia AJ, Daviglus ML, Dyer AR, et al. (1996) Fish consumption and stroke in men: 30-year findings of the Chicago Western Electric Study. Stroke 27, 204-209.

37. Yang G, Shu XO, Jin F, et al. (2004) Soyfood consumption and risk of glycosuria: a cross-sectional study within the Shanghai women's health study. Eur J Clin Nutr 58, 615-620.

38. Wennberg M, Bergdahl IA, Stegmayr B, et al. (2007) Fish intake, mercury, long-chain $n-3$ polyunsaturated fatty acids and risk of stroke in northern Sweden. Br J Nutr 98, 1038-1045.

39. Willett W (1998) Nutritional Epidemiology. New York: Oxford University Press. 